\title{
Intranasal Delivery of Whole Cells of Neisseria Species: Study of Cross - Reactive Antigens in Rabbits
}

\author{
Claudia F. Tunes ${ }^{1}$, Aline S. Ferraz ${ }^{1}$, Monica C.G. Scola ${ }^{2}$ and Elizabeth N. De Gaspari*,1 \\ ${ }^{1}$ Imunology Section Adolfo Lutz Institute, São Paulo, SP, Brazil and ${ }^{2}$ Bacterial Culture Collection, Adolfo Lutz Institute, \\ São Paulo, São Paulo, Brazil
}

\begin{abstract}
Neisseria lactamica, a commensal bacterium that is non-pathogenic to human beings and that is usually found in the upper respiratory tract of children, is closely related to the pathogenic species Neisseria meningitidis. A protocol was proposed to study the immunogenicity of killed whole cells of $N$. lactamica, $N$. meningitidis, $N$. sicca or $N$. meningitidis c (carrier-isolated) by i.n. immunization in rabbits,considering the natural pathogen entry route. Adult rabbits were inoculated i.n with oropharynx-isolated $N$. lactamica, $N$. meningitidis, $N$. sicca or $N$. meningitidis carrier strain (c), at a concentration of 1.0 optical density unit at $650 \mathrm{~nm}$ in a volume of $500 \mu \mathrm{L}$. The rabbits were immunized four times at sevendays intervals. The rabbits developed levels of specific IgG antibodies in serum, as determined by ELISA using whole cells of homologous and heterologous strains. Serum from rabbits immunized with $N$. lactamica, $N$. meningitidis, and $N$. sicca or $N$. meningitidis c strain, showed IgG antibodies reactive to 5 to $190 \mathrm{kDa}$ antigens on immunoblotting with high avidity.
\end{abstract}

Key Words: N. lactamica, N. meningitidis, rabbits, nasal immunization, humoral immune response, cross-reactive antigens.

\section{INTRODUCTION}

Invasive disease caused by Neisseria meningitidis represents a significant global public health problem, and its prevention by vaccination remains a high priority worldwide.

N. meningitidis, a gram-negative, encapsulated bacterium, is one of the major causative agents of bacterial meningitis in humans. Effective vaccines based on capsular polysaccharides are available for disease caused by serogroups A, $\mathrm{C}$ Y and W135 meningococci, but the protection afforded by polysaccharide vaccines is time-limited and very poor in the younger children [1].

Conjugate polysaccharide vaccines that provide improved immunogenicity in young children are becoming available. The polysaccharide-based vaccines for serogroup B $N$. meningitidis have failed to induce an effective immune response; this failure appears to be due to the structural similarity of the polysialic acid chains of group B capsular polysaccharide to host polysialylated glycoproteins such as neural cell adhesion molecule [1].

Efforts to produce an efficacious vaccine against serogroup B $N$. meningitidis have met with only moderate success. Outer membrane protein-based vaccines have demonstrated more effective serum bactericidal antibody response; furthermore, it could be stimulated if the proteins were in a more native conformation, without any detergent treatment or extraction of lipooligosaccharide (LOS) [2]. Nevertheless, some studies have pointed to the possibility that the use of OMP-based vaccines of $N$. meningitidis could lead to several problems, such as the diminution of carrier rates or the hampering of colonization by related commensal species,

*Address correspondence to this author at the Imunology Section Adolfo Lutz Institute, São Paulo, SP, Brazil; Tel: 55 (11) 3068-2898; Fax: 55 (11) 3068-3505; E-mail: egaspari@ial.sp.gov.br such as $N$. lactamica, that share common OMP antigens with the meningococci [3].

Serogroup B meningococcal vaccines are based on outer membrane vesicles (OMVs) containing major and/or important proteins, such as class proteins (PorA, PorB, Opa), transferring-binding proteins (Tbps), ferric binding proteins A ( $\mathrm{FbpA})$, and some others. Although relatively efficacious, the OMV vaccines are usually very specific against the strains from which they are developed; thus, efforts are currently focused on the search for immunogenic proteins showing broad cross-reactivity [4].

Protective antibodies against $N$. meningitidis are acquired progressively over the first few years of life, a period when meningococcal carriage rates are low but carriage rates of commensal Neisseria are higher. Thus, it has been postulated that the acquisition of natural immunity in children may follow colonization of the nasopharynx by non-pathogenic species of Neisseria and some other bacteria expressing immunologically cross-reactive surface antigens shared with meningococci [5].

Neisseria lactamica, a commensal species that does not possess a polysaccharide capsule or PorA but shares many common antigens with $N$. meningitidis may be the most important of these [6]. However, there are studies that have shown that the inclusion of such cross-reactive proteins in a vaccine may be lead to consequences in the colonization by $N$. lactamica, interfering in the development of natural immunity [7].

Previous studies have shown that OMVs from N. lactamica are an alternative approach toward a meningococcal vaccine [8]. The induction of a local mucosal response to supplement the systemic response may lead to more effective immunization against pathogenic meningococci. Intranasal (i.n) immunization would be a direct approach to stimulating 
a mucosal response, since the human nasopharyngeal region is the natural habitat for meningococci and it is believed that nasopharyngeal carriage leads to natural immunization [9].

Several studies show that meningococcal native outer membrane vesicles (NOMV) can be safely used as an intranasal vaccine based on findings in human volunteers, and that this induces a high quality antibody response characterized by persistent serum antibodies with bactericidal activities and also both a mucosal and a serum antibody response [10]. A recent study using the rabbit model demonstrated that intranasally administered meningococcal NOMV induced a good mucosal response in the rabbit, indicated by the presence of $\operatorname{IgA}$ in nasal wash and lung lavage fluid; in contrast, there was no evidence of mucosal IgA production with the samples obtained from animals immunized intramuscularly [2]. The rabbit model to show a more robust response to intranasal vaccination humans; and to be more consistent in their antibody response compared to mice. This was observed in a study by Shoemaker et al. [2] who found a wide range of antigens (as determined by Western blotting) that were induced by intranasal vaccination in rabbits, and this was a significant response to the LOS component of the NOMV. Besides this, it is easy to vaccinate unanesthetized rabbits intranasally and thereby avoid the problem of vaccine entering the lung [2].

In a study performed by Oliver et al. [9], it was observed that immunization with killed $N$. lactamica whole cells, outer membrane vesicles (OMV) or outer membrane protein (OMP) protected mice against lethal challenge by various serogroup $\mathrm{B}$ and $\mathrm{C}$ meningococcal isolates in a model of bacteremic infection, although no correlation with bactericidal activity occurred, indicating that other mechanisms may be involved in this protection. Opsonophagocytosis activity mediated by antibody response has been observed in rabbit models with the administration of $N$. lactamica OMV vaccine, indicating that this mechanism of protection may be involved [7]. Indeed, the protection in the absence of bactericidal activity can be attributed to other antigens such as transferring binding protein A (TbpA) [9], the genomederived vaccine candidate 2132 [11] or the meningococcal vaccine candidate NspA (expressed in OMV from $N$. flavences recombinant bacteria, a commensal Neisseria species), where protection and opsonophagocytosis activity in a mouse model of meningococcal bacteremia were observed [12].

The protective activity of purified $N$. lactamica LOS is not clear, but it is possible that the experimental $N$. lactamica vaccines, containing LOS in a more native and immunogenic form, can offer protection [9].

Various studies show that $N$. lactamica OMV are similarly immunogenic compared to $N$. meningitidis OMV when delivered intranasally. Moreover, $N$. lactamica OMV can elicit cross-reactive immune responses with $N$. meningitidis and act as an effective intranasal adjuvant [8]. In a recent study, Li Y et al. [13], demonstrated experimentally that the immunization of mice with live $N$. lactamica protects animals against lethal meningococcal challenge, and that some but not all strains of $N$. lactamica elicit detectable serum bactericidal activity (SBA) in immunized animals, regardless of the serogroup of $N$. meningitidis. However, it unlikely that immunization with live $N$. lactamica will be implemented as a vaccine against meningococcal disease, but the knowledge of the induction of cross-protective immunity and SBA can help substantially in developing sub-unit vaccines against this important infectious disease.

A protocol was proposed to study the immunogenicity of Neisseria species and $N$. meningitidis $\mathrm{B}$ in the rabbit model considering the natural pathogen entry route, and the potential of these antigens for the development of a vaccine against meningococcal disease. We therefore developed an alternative animal model using the rabbit; this model more closely mimics intranasal immunization of humans.

The aim of the present study was to determine the specificity and ability of antibody protection through bactericidal activity and avidity induced in rabbits immunized intranasally with killed whole cells of commensal strains, N. meningitidis $c$ (carrier isolated) and $N$. meningitidis $B$.

\section{MATERIALS AND METHODS}

\section{Rabbits}

Twenty-four adult male New Zealand white rabbits (CRIEX Laboratories-São Paulo - SP- Brazil) weighing 2.25 to $3.5 \mathrm{~kg}$ were used in all experiments. We used 3 rabbits for each experimental group. Intranasal immunization was accomplished with unanesthetized rabbits by using a twoperson procedure. Rabbits were held in a supine position and a flexible micropipettor was used to drip $500 \mu \mathrm{L}$ of vaccine in the nares, with about half the volume in each nares. Research was conducted in compliance with the principles stated in the Brazilian Code for the Care and Use of Laboratory Animals and other federal statutes and regulations relating to animals and experiments involving animals CTC-IAL\# 03/03.

\section{Bacterial Strains}

The N. meningitidis B:4,7:P1.15,19 (44/89) strain was isolated from a patient with meningococcal disease in Greater São Paulo. N. lactamica strains were isolated from the oropharynx or of healthy carriers in our community and $N$. lactamica, $N$. subflava, $N$. perflava, $N$. elongata, $N$. sicca and $N$. mucosa strains were isolated from blood or cerebrospinal fluid of patients with meningococcal disease, which were obtained from the reference center Adolfo Lutz Institute (São Paulo- Brazil). Strains of $N$. lactamica, $N$. sicca and $N$. meningitidis obtained from the oropharynx of carriers were also isolated, and these strains were kindly provided by Dr. Ferreirós of Departament of Microbiology, Faculty of Pharmacy, University of Santiago of Compostela, Spain.All bacterial stocks were kept at $-70^{\circ} \mathrm{C}$ in skim milk solution or were lyophilized and stored at $4{ }^{\circ} \mathrm{C}$. $N$. meningitidis and Neisseria species were grown overnight in a candle jar in tryptic soy broth (TSB- Difco BRL Products, Gaithersburg, MD) supplemented with $1 \%$ horse serum (Sigma, St. Louis, $\mathrm{MO}$ ) in plates in a $5 \% \mathrm{CO}_{2}$ atmosphere at $37^{\circ} \mathrm{C}$. For the assays used, the whole cell strains of $N$. meningitidis and Neisseria species were suspended in phosphate-buffered saline (PBS), $\mathrm{pH} 7.4$, containing $0.02 \%$ sodium azide and heat killed. The absorbance of the suspensions was adjusted to 0.1 O.D. at $650 \mathrm{~nm}$ using a CELM - E2250 Spectrophotometer (Table 1). 
Table 1. Characteristics of the Neisseria Strains Used in This Study

\begin{tabular}{|c|c|}
\hline Strain & Origin \\
\hline \multicolumn{2}{|c|}{ N. meningitidis B epidemic strain } \\
\hline $\mathrm{N} 44$ / 89 & CSF \\
\hline N $360 / 88$ & CSF \\
\hline \multicolumn{2}{|c|}{ N. meningitidis $\mathrm{B}$ isolated carrier } \\
\hline N 391 (A:P1.9) (Spain) & Oropharynx \\
\hline \multicolumn{2}{|l|}{ N. subflava subflava } \\
\hline N $456 / 99$ & CSF \\
\hline \multicolumn{2}{|l|}{ N. subflava perflava } \\
\hline N $613 / 98$ & $\mathrm{CSF}$ \\
\hline $\mathrm{N} 20 / 00$ & $\mathrm{CSF}$ \\
\hline N $148 / 00$ & CSF \\
\hline \multicolumn{2}{|l|}{ N. elongata } \\
\hline N $699 / 99$ & CSF \\
\hline \multicolumn{2}{|l|}{ N. subflava flava } \\
\hline N $123 / 00$ & $\mathrm{CSF}$ \\
\hline \multicolumn{2}{|l|}{ N. mucosa } \\
\hline $\mathrm{N} 44 / 00$ & CSF \\
\hline \multicolumn{2}{|l|}{ N. sicca } \\
\hline N $926 / 99$ & Blood \\
\hline NSP10 (Spain) & Oropharynx \\
\hline \multicolumn{2}{|l|}{ N. lactamica } \\
\hline N $799 / 98$ & $\mathrm{CSF}$ \\
\hline N $1398 / 00$ & Oropharynx \\
\hline N 831/98 & Oropharynx \\
\hline N 844/95 & Oropharynx \\
\hline N $602 / 91$ & Oropharynx \\
\hline N $362 / 92$ & Oropharynx \\
\hline N $852 / 93$ & Oropharynx \\
\hline N $952 / 93$ & Oropharynx \\
\hline N 1024 / 94 & Oropharynx \\
\hline N 924 / 93 & Oropharynx \\
\hline N $124 / 94$ & Oropharynx \\
\hline NIP4 (Spain) & Oropharynx \\
\hline
\end{tabular}

CSF: cerebrospinal fluid.

\section{Isolation of Native Outer Membrane Vesicles (NOMV)}

NOMVs from the strain N. meningitidis B: 4,7: P1.15,19 (44/89) were obtained as described by Gomez et al. [14].
Briefly, bacteria from five plates were recovered by centrifugation at $10,000 \times \mathrm{g}$ for $10 \mathrm{~min}$ at $4^{\circ} \mathrm{C}$ and suspended in 0.1 $\mathrm{M}$ acetate buffer, pH 5.8 , with $0.2 \mathrm{M} \mathrm{LiCl}$ ( $5 \mathrm{ml} / \mathrm{g}$ of cells) and incubated for $2 \mathrm{~h}$ at $45^{\circ} \mathrm{C}$ in a shaking water bath with 6 $\mathrm{mm}$ glass beads at $300 \mathrm{rpm}$. The supernatant was recovered by centrifugation $\left(10,000 \times \mathrm{g}, 10 \mathrm{~min} ., 4^{\circ} \mathrm{C}\right)$. The preparation was diluted to a final concentration of $1 \mathrm{mg} / \mathrm{mL}$ and stored in aliquots at $-70^{\circ} \mathrm{C}$. Protein content was determined by the method described by Lowry [15].

\section{Immune Sera}

The rabbit immune sera were obtained using killed whole cells of $N$. meningitidis, $N$. lactamica, $N$. sicca or carrierisolated $N$. meningitidis preparations previously selected by 8C7Br1 Mab described by De Gaspari (2000) [16] from cultures and following the immunization protocol described below (Table 2 ).

\section{Immunization Protocol}

The rabbits were immunized four times at seven-day intervals; the whole cells were administered intranasally by instillation in the nares, at a concentration of 1.0 optical density at $650 \mathrm{~nm}$ in a volume of $500 \mu \mathrm{L}$ of vaccine, in the absence of adjuvants. Blood was collected from the central ear vein on day 0 (prior to inoculation), and days 7, 14, 21 and 35 (1 week following each inoculation).

\section{SDS-PAGE and Immunoblot Analysis}

Whole cells of $N$. meningitidis (44/89), N. meningitidis $c$, $N$. lactamica, $N$. sicca, $N$. subflava, N. perflava, N. elongata, $N$. mucosa strains and NOMVs of $N$. meningitidis (44/89) used in this study were digested in SDS and resolved by electrophoresis on SDS slab gels according to the method described by Laemmli [17] with some modifications. A $13 \%$ acrylamide resolving gel (Sigma), and a 4\% stacking gel were used. Samples were prepared by mixing one part whole cells (O.D. of 1.0 at $650 \mathrm{~nm}$ ) with one part sample buffer (62.5 mM Tris-HCl, pH 6.8, 1\% (v/v) glycerol, 2\% (w/v) SDS, $2 \%$ mercaptoethanol, and $0.5 \%(\mathrm{w} / \mathrm{v})$ bromophenol blue, and heated for $5 \mathrm{~min}$ at $100^{\circ} \mathrm{C}$. Samples $(25 \mu \mathrm{L})$ were applied to each gel lane. Electrophoresis was carried out at $50-\mathrm{V}$ constant voltage. Protein standards (Pharmacia LKB, Uppsala, Sweden) were used for determination of apparent molecular weight. The protein bands were detected by staining with Coomassie Brilliant Blue (Pharmacia LKB, Biotechnology, Piscataway, NJ, USA). After SDS-PAGE, proteins were transferred electrophoretically from the gel to a nitrocellulose membrane (BioRad), according to the method of Towbin et al. [18]. A constant potential of $5 \mathrm{v} / \mathrm{cm}$ was applied to gel-nitrocellulose membrane sandwich for $18 \mathrm{~h}$ in an electroblotter system (BioRad) as specified by the manufacturer in an electroblotting buffer containing $25 \mathrm{mM}$ Tris$\mathrm{HCl}, 192 \mathrm{mM}$ glycine, and 20\% (v/v) methanol, pH 8.3. After assessing transfer efficiency by Ponceau-S staining, the membranes were blocked with 5\% skim milk (Gloria-Brazil) in PBS for $2 \mathrm{~h}$ at room temperature to block nonspecific protein-binding sites, washed five times with PBS, $\mathrm{pH} 7.4$, and then incubated overnight at $37^{\circ} \mathrm{C}$ with a previously determined working dilution of the immune sera of rabbits (1:500 in all cases). One day later, the membranes were washed again and were then incubated with HRP-conjugated rabbit anti-goat immunoglobulin IgG (Sigma Chemical Company, 
Table 2. Immunization Protocol for Obtaining the Different Sera Against Neisserial Inactivate whole Cells by Intranasal Route. The Rabbits were Immunized as the A Concentration of Optical Density 1.0 at $650 \mathrm{~nm}$ in a Volume of $500 \mu \mathrm{L}$

\begin{tabular}{|c|c|c|c|c|c|}
\hline Sera & Day 0 (1 st Dose) & Day 7 (2 nd Dose) & Day 14 (3 rd Dose) & Day 21 (4 th Dose) & Day 35 \\
\hline \hline NL & NL & NL & NL & Notal bled \\
\hline NLNM & NL & NL & NS & NS & NM \\
\hline NS & NS & NS & NM & Nc \\
\hline NSNM & NS & NS & NM & NM \\
\hline Nc & Nc & Nc & NM & NL & NL \\
\hline NM & NM & NM & NM & N & \\
\hline
\end{tabular}

(NL) N. lactamica strain 1024/94 (Brazil); (NM) N. meningitidis strain 44/89 (Brazil); (NS) N. sicca strain NSP10 (Spain); (Nc) N. meningitidis isolated-carrier strain P.391 (A:P1.9). (Spain).

St. Louis, MO). After $2 \mathrm{~h}$ incubation and four washes, the blots were soaked in a solution of $5 \mu \mathrm{L} \mathrm{H}_{2} \mathrm{O}_{2}(30 \%)$, and developed with 3-amino-9-ethylcarbazole (AEC) (Pierce).

\section{ELISA}

The method was performed to determine IgG levels based on the method described by Abdillahi \& Poolman [19]. Detection of rabbit IgG was carried out using flatbottom microtiter plates (Polysorb - Costar, Cambridge, MA). Aliquots of $100 \mu \mathrm{L}$ of bacterial suspension were used to coat the wells of $96-w e l l$ plates for $18 \mathrm{~h}$ at $37^{\circ} \mathrm{C}$. The suspensions of bacteria were prepared to an optical density of 0.1 at $650 \mathrm{~nm}$ in PBS containing $0.02 \%$ sodium azide. The plates were washed once with PBS- $0.05 \%$ Tween and blocked with $200 \mu \mathrm{L}$ of $5 \%$ skim milk (Gloria-Brazil) in PBS for $2 \mathrm{~h}$ at $37^{\circ} \mathrm{C}$. The plates were washed five times with PBS-0.05\% Tween, incubated with $100 \mu \mathrm{L}$ per well of serial twofold dilutions of test sera for $2 \mathrm{~h}$ at $37^{\circ} \mathrm{C}$, and washed once with PBS-0.05\% Tween. The plates were then incubated with HRP-conjugated goat anti-rabbit immunoglobulin IgG (Sigma Chemical Company, St. Louis, MO) diluted at 1:5000 in PBS for $2 \mathrm{~h}$ at $37^{\circ} \mathrm{C}$. The wells were washed again and antibodies quantified by developing with $100 \mu \mathrm{L}$ of 3,3',5,5'-tetramethylbenzidine (TMB) for $20 \mathrm{~min}$. Sulfuric acid $(1 \mathrm{~N}, 100 \mu \mathrm{L})$ was added to each well to stop the reaction, and absorbance was read at $450 \mathrm{~nm}$ in a microtiter plate reader. The antibody titer was determined as the reciprocal dilution of serum corresponding to the midpoint of the doseresponse curve. The cut-off was determined as being the greater value of the antibodies present in the pool of preimmune sera. For the immune sera, reactivity was considered for all the values above this reactivity threshold.

\section{Bactericidal Assay}

The bactericidal assay was performed using Brazilian epidemic serogroup B strain $N$. meningitidis B: 4,7: P1.15,19 (44/89) in 96-well plates, as described previously by Mandrell et al. [20], with some modifications. Briefly, the final reaction mixture $(50 \mu \mathrm{L})$ contained $25 \mu \mathrm{L}$ of serial twofold dilutions of test serum that had been heat-inactivated at $56^{\circ} \mathrm{C}$ for $30 \mathrm{~min}, 12.5 \mu \mathrm{L}$ guinea pig serum as the complement source, screened for the absence of antimeningococcal activity, and $12.5 \mu \mathrm{L}$ of a suspension of exponential-phase meningococci $\left(3 \times 10^{3} \mathrm{CFU} / \mathrm{mL}\right.$ per well), grown in brain heart infusion agar (BHI-Difco) containing $1.0 \%(\mathrm{v} / \mathrm{v})$ horse serum. The reaction mixture was incubated at $37^{\circ} \mathrm{C}$ for $30 \mathrm{~min}$ as described by Maslanka et al. [21]. A volume of $130 \mu \mathrm{L}$ tryptic soy broth, (TSB-Difco) containing $0.9 \%$ (w/v) Noble (Difco) agar and a mixture of vancomycin, colistin and nystatin (VCN-Laborclin) at $0.15 \%(\mathrm{v} / \mathrm{v})$, was added to each well. The mixture was allowed to solidify, and the plates were incubated for $18 \mathrm{~h}$ at $37^{\circ} \mathrm{C}$ in $5 \% \mathrm{CO}_{2}$. Viable counts were recorded at time $0\left(t_{0}\right)$, before incubation with complement, in order to estimate the number of CFU for $100 \%$ survival, by plating $12.5 \mu \mathrm{L}$ bacterial stock suspension onto a $150 \times 150 \mathrm{~mm}$ TSB plate. Control wells included in each microtiter plate contained: (i) bacteria, complement and buffer (complement-dependent control) and (ii) bacteria, heat-inactivated complement and buffer (complementindependent control). In addition, a positive control was included in each assay consisting of serial dilutions of a known positive serum. Assays were performed in duplicate. The bactericidal titer of the serum was defined as the reciprocal of the serum dilution giving $\geq 50 \%$ killing of the bacteria present at $t_{0}$.

\section{High-Avidity Antibodies}

We performed a modified immunoglobulin $\mathrm{G}$ (IgG) ELISA to measure concentrations of IgG antibodies in the test serum directed against whole cells of $N$. lactamica, $N$. meningitidis, $N$. sicca or $N$. meningitidis $c$ (carrier-isolated) strains, as described by Granoff et al. [22]. The only modification resulted in an IgG ELISA performed with and without $75 \mathrm{mM} \mathrm{NH}_{4} \mathrm{SCN}$ in the serum diluting buffer in an attempt to select high-avidity antibody binding. Ammonium thiocyanate is a chaotropic agent which inhibits antigen-antibody interactions in a dose-dependent fashion. The avidity of antibodies was defined as the average ratio of absorbance in the presence of SCN and in the absence of SCN. (An avidity index value less than 0.29 was designated low avidity, 0.30- 
0.49 intermediate avidity, and greater than 0.49 high avidity) [23].

\section{RESULTS}

\section{Immune Response}

The systemic immune response of rabbits was determined by measuring meningococcus-specifc $\operatorname{IgG}$ titers in serum after intranasal administration of whole cells of bacterial suspension prepared from commensal strains or N. meningitidis (Table 1). The rabbits were immunized in the absence of adjuvants four times at seven-day intervals and the sera obtained were analyzed individually and assayed on the $35^{\text {th }}$ day after immunization. The titers of $\operatorname{IgG}$ antibodies reached were dependent of the immunization scheme and bacterial concentration used in the assay (Table 2), which allowed us to obtain sera with relatively high antibody titers. Mean titers of the NL sera and that of the NM serum were $1: 8000$ and 1:4000, respectively, when determined against whole cells from the homologous strains, and varied from 1:4000 to 1:2000 when determined against heterologous strains. Antibodies induced against whole cells of $N$. lactamica showed a high degree of cross-reactivity with $N$. meningitidis and commensal strains when determined by ELISA and immunoblotting. Coomassie Blue-stained polyacrylamide gels confirmed a similar banding pattern of proteins among these strains and also with NOMV of N. meningitidis 44/89 strain (Fig. 1). The bactericidal activity of each serum was assessed against the meningococcal strain 44/89. Bactericidal activity was relatively low in sera raised against $N$. lactamica whole cells, but can it be considered positive according to the criteria established ( $\geq 50 \%$ killing). Bactericidal activity was increased for the NLNM sera of immunization scheme to a mean titer of $1 / 128$, which is considered statistically significant but is not sufficient. Only rabbit serum raised against $N$. meningitidis strain showed strong in- vitro bactericidal activity (1/2048) towards these strains (Table 3). High avidity $\lg G$ antibodies were produced for all immunization schemes, although significant correlation was not found among ELISA titers, bactericidal activities and induction of $\operatorname{lgG}$ antibodies of high avidity obtained for all the sera against that test strain, mainly in rabbits immunized with $N$. lactamica (Table 3).

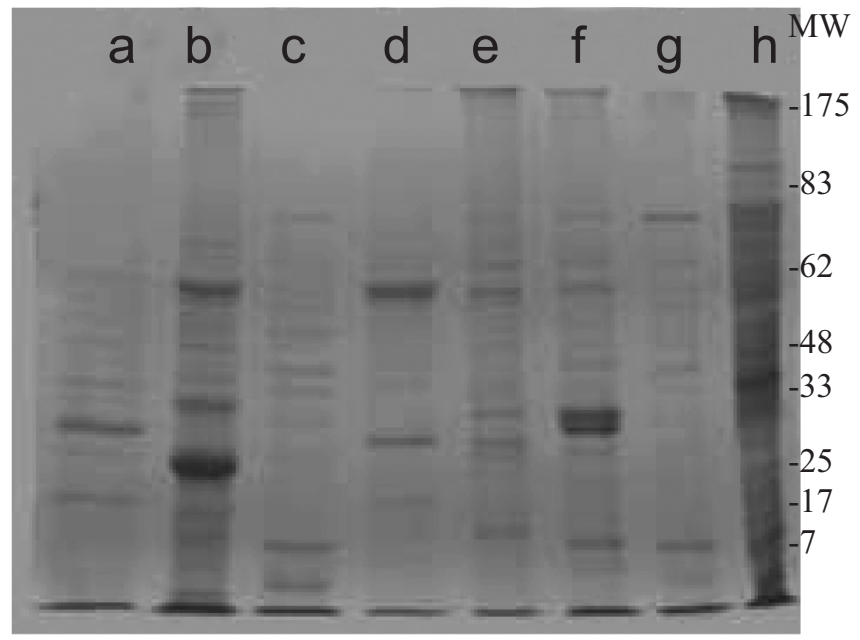

Fig. (1). Commassie blue-stained polyacrylamide gel (SDS-PAGE) from the strains $N$. meningitidis, Neisseria species whole cells and NOMV of $N$. meningitidis strain. a) NOMV of strain 44/89 NM, b) whole cell of strain 44/89 NM, c) whole cell of strain 360/ $88 \mathrm{NM}$, d) whole cell of strain 1024 / $94 \mathrm{NL}$, e) whole cell of strain 602 / 91NL, f) whole cell of strain 799 / $98 \mathrm{NL}, \mathbf{g}$ ) whole cell of strain NSP10 NS, h) whole cell of strain $391 \mathrm{NMc}$. (NM) N. meningitidis; (NL) N. lactamica; (NS) N. sicca; (NMc) N. meningitidis isolatedcarrier. MW (Pattern of molecular weights are on the right-hand side).

Table 3. Bactericidal Activity, ELISA Antibody Titers and Avidity Antibodies of Rabbit Sera Used

\begin{tabular}{|c|c|c|c|c|c|c|}
\hline Sera & NM & NL & NS & Nc & Bactericidal Activity Titers* & Avidity Antibodies** \\
\hline NL & $1: 4000$ & $1: 8000$ & $1: 4000$ & $1: 4000$ & $1 / 4$ & 0.37 \\
\hline NLNM & $1: 2000$ & $1: 4000$ & $1: 4000$ & $1: 4000$ & $1 / 128$ & $0.75-1.00$ \\
\hline NSNM & $1: 4000$ & $1: 4000$ & $1: 2000$ & $1: 2000$ & $1 / 32$ & 0.76 \\
\hline $\mathrm{Nc}$ & $1: 2000$ & $1: 4000$ & $1: 4000$ & $1: 8000$ & $1 / 128$ & 1.00 \\
\hline $\mathrm{NcNM}$ & $1: 4000$ & $1: 2000$ & $1: 2000$ & $1: 4000$ & $1 / 256$ & 0.54 \\
\hline NM & $1: 4000$ & $1: 2000$ & $1: 2000$ & $1: 2000$ & $1 / 2048$ & 0.75 \\
\hline
\end{tabular}

(NL) N. lactamica strain 1024/94 (Brazil); (NM) N. meningitidis strain 44/89 (Brazil); (NS) N. sicca strain NSP10 (Spain); (Nc) N. meningitidis isolated-carrier strain P.391 (A:P1.9). (Spain).

*Bactericidal activity and avidity antibodies parameters were both determined using strain N. meningitidis $44 / 89$ for testing. In the study we utilize a medium of Three independent experiments.

**(Avidity index values less than 0.29 was designated the low avidity; 0.30-0.49 the intermediate avidity and greater than 0.50 the high avidity).

*** end point ELISA titers.

We used the medium of 3 rabbits serum for each experiment. 


\section{Cross-Reactive Antigens}

Analysis of the specificity of the antibody response of rabbits to intranasal vaccination with whole cells of commensal strains or $N$. meningitidis was carried out by immunoblotting using whole-cell bacterial suspension vaccine and OMV of $N$. meningitis (44/89) strain as antigens. All the strains used in our study were previously selected by $8 \mathrm{C} 7 \mathrm{Br} 1$ Mab as described by De Gaspari (2000) [16]. The results of immunoblotting revealed that intranasal vaccination caused an antibody response against a broad range of outer membrane antigens, including both outer membrane proteins and LOS. As seen in Fig. (2), major responses are elicited against 50-60, 70-80 and $190 \mathrm{kDa}$ antigens; however, a correlation among responses to a particular antigen (or a group of antigens) in immunoblotting (Fig. 2), ELISA IgG titers and bactericidal activity was not observed, mainly when the immu- nization scheme of four doses of $N$. lactamica was used (Table 3).

\section{DISCUSSION}

In this study, we demonstrated and determined the specificity and ability of antibody protection through bactericidal activity and the degree of avidity induced in rabbits immunized intranasally with whole cells of $N$. lactamica, $N$. sicca, $N$. meningitidis and N. meningitidis (carrier-isolated), in an attempt to correlate ELISA IgG titers, bactericidal activities and induction of $\operatorname{lgG}$ antibodies of high avidity obtained for all the rabbit sera after scheme immunization, and also to evaluate the improvement of the immune response of mucosal as well as systemic immunity by exploring the immunogenicity of N. lactamica and its capacity to induce crossreactive antibodies with $N$. meningitidis and other Neisseria

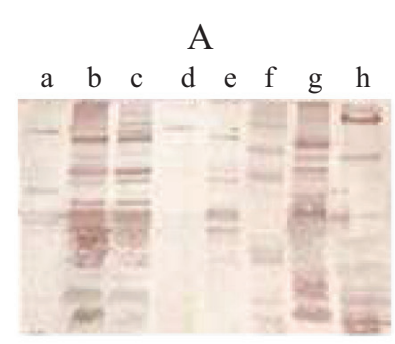

C

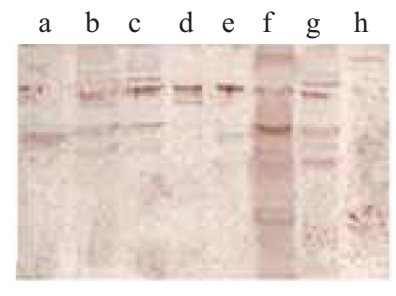

$\mathrm{E}$

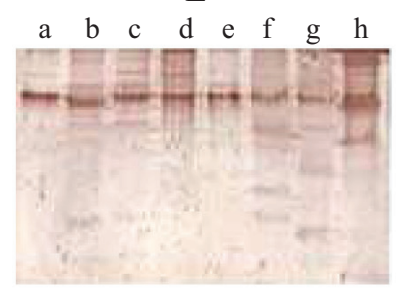

$\mathrm{G}$

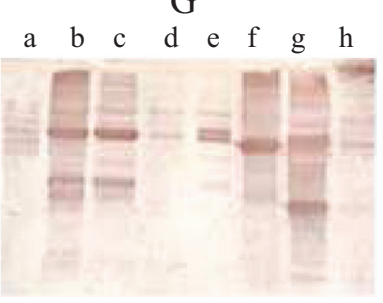

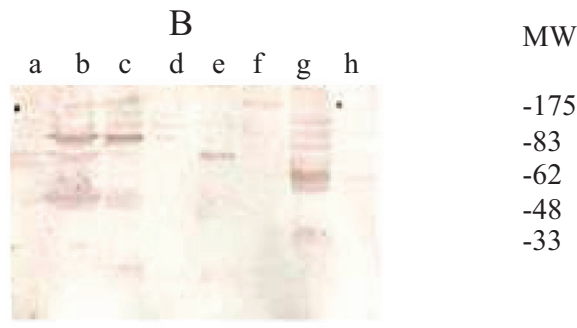

$\mathrm{D}$
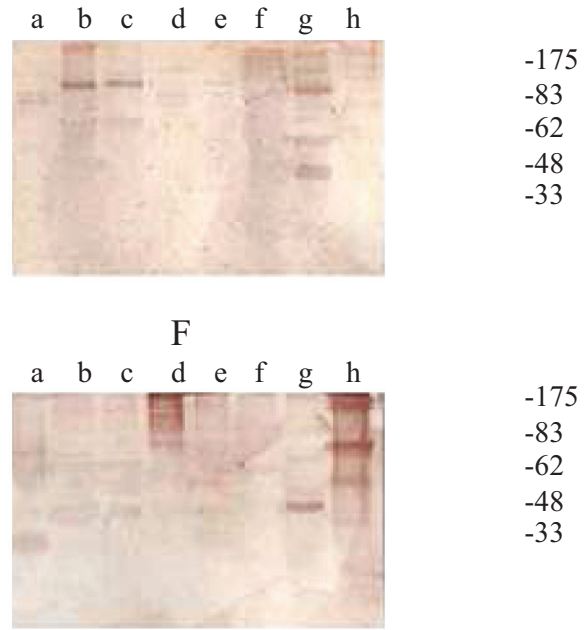

$\mathrm{H}$

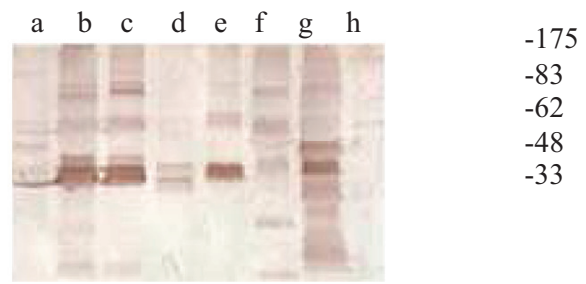

Fig. (2). Immunoblot analysis of whole cells antigens from the strains $N$. meningitidis, Neisseria species and NOMV of $N$. meningitidis strain. The sera pool of three rabbits were analyzed and measured on the $35^{\text {th }}$ day after immunization. The strains used in, a) NOMV of $44 / 89$ NM strain, b) whole cell of 44/89 NM strain, c) whole cell of 360 / 88 NM strain, d) whole cell of 1024/ 94 NL strain,e) whole cell of 602 / 91NL strain, f) whole cell of 799 / 98 NL strain, $\mathbf{g}$ ) whole cell of NSP10 NS strain, h) whole cell of 391 NMc strain. The Immunoblot cam be analysed with the pool sera of three rabits. In A) sera of rabbits immunized with NL, B) sera of rabbits immunized with NLNM, C) sera rabbits immunized with NS, D) sera of rabbits immunized with NSNM, E) sera of rabbits immunized with Nc, F) sera of rabbits immunized with NcNM, G) sera of rabbits immunized with NM, H) sera of rabbits immunized with NMNL. (NM) N. meningitidis; (NL) N. lactamica; (NS) N. sicca; (Nc) N. meningitidis isolated-carrier. MW (Pattern of molecular weights are on the right-hand side.). 
species. We therefore developed an alternative animal model using the rabbit; compared to the murine model, this model more closely mimics intranasal immunization of humans for vaccine tests. The rabbits used are considered an outbred population and can reproduce responses with a high degree of heterogeneity corresponding to responses in vaccinated humans.

In recent years, intranasal immunization has received considerable attention as a potentially effective, noninvasive method of vaccination. Mucosal vaccines are easy to deliver and have the potential of conferring protection at the site of infection [7].

Various studies have been conducted using rabbit models involving intranasal immunization. Suckow et al. (1996) [24] studied the use of cholera toxin as mucosal adjuvant in the immunization of rabbits against Pasteurella multocida, and they observed more protection as compared to animals that received no co-adjuvant. In a study of passive transfer in rabbits and mice described by Lu (1991) [25], a monoclonal antibody was tested as a vaccine against $P$. multocida outer membrane proteins, where protection was observed after challenge with homologous and heterologous strains, and this protection was antigen specific. Shoemaker et al. (2005) [2] showed that intranasal immunization in rabbits with meningococcal serogroup B NOMV induced local mucosal and serum bactericidal antibody responses; furthermore, they could easily vaccinate unanesthetized rabbits intranasally and thereby avoid the problem of vaccine entering the lung.

Carriage of commensal Neisseria spp, particularly N. lactamica, has been considered important for the development and maintenance of natural immunity to meningococcal disease in young children, and both antibody cross-reactivity and bactericidal antibodies to meningococci have been determined following $N$. lactamica carriage in humans [3].

OMV vaccines for serogroup B have demonstrated efficacy in older children, but protection does not occur in young children. Indeed, the OMV vaccines are usually very specific against the strains from which they have been derived; thus, current interest is focused on the search for immunogenic proteins showing broad cross-reactivity [4].

Neisseria lactamica, a commensal species that does not possess a polysaccharide capsule or PorA but shares many common antigens with $N$. meningitidis, may be the most important organism for studies of protection against meningococcal disease [9].

Our results demonstrated the presence of common and conserved antigens between strains of $N$. meningitidis and Neisseria species related principally within a range of 50-60 $\mathrm{kDa}$ proteins when they were analyzed with $8 \mathrm{C} 7 \mathrm{Br} 1 \mathrm{Mab}$ by immunoblotting. The $8 \mathrm{C} 7 \mathrm{Br} 1 \mathrm{Mab}$ previously described by De Gaspari (2000) [16] was used to select all the strains that expressed the $50-65 \mathrm{kDa}$ protein on membrane surfaces in our study (data not shown).

The $8 \mathrm{C} 7 \mathrm{Br} 1 \mathrm{MAb}$ also reacted in a range of $50-65 \mathrm{kDa}$ proteins with $70 \%$ or more of $N$. lactamica strains used in the study ( data not shown). We also analyzed the reactivity and specificity pattern of the immune sera obtained from rabbits immunized intranasally with whole cells of a bacterial suspension prepared from $N$. lactamica, N. meningitidis,
$N$. sicca or $N$. meningitidis $c$, using immunoblotting. In this immunoblot, an antibody response against a broad range of outer membrane antigens, including both outer membrane proteins and LOS, was demonstrated. Patterns of homologous and heterologous reactivity among the strains analyzed with major responses directed against 50-60, 70-80 and 190 $\mathrm{kDa}$ antigens in immunoblotting, were also revealed, although a correlation between response to a particular antigen (or a group of antigens) and bactericidal activity was not observed. The specificity of $8 \mathrm{C} 7 \mathrm{Br} 1$ with $50 \mathrm{kDa}$ proteins in the blot suggests that the sharing of common antigens among strains of $N$. meningitidis, $N$. lactamica and other Neisseria species used in this study in the 50-65 kDa range, can be considered promising for a new formulation of a vaccine against meningococcal serogroup B.

Recently, a group that has been working for more than 15 years on the characterization of protective antigens isolated from $N$. lactamica neisseria species described the use of a polyclonal serum to detect a new protein of $47 \mathrm{kDa}$ present in N. meningitidis. Protein P47 was identified by MALDITOF fingerprinting analysis as the hypothetical lipoprotein NMB0035 [26]. Western blotting with the polyclonal monospecific serum showed that linear P47 epitopes were expressed in similar amounts in the Neisseria meningitidis strains tested and, to a lesser extent, in commensal Neisseria, particularly $N$. lactamica. These investigators suggested that this antigen is also important in vaccine preparation.

We performed an ELISA with the aim of evaluating the systemic immune response of rabbits immunized intranasally with the strains used in this study, through the determination of $\mathrm{IgG}$ antibody titers produced in rabbits, in an attempt to find a correlation between bactericidal activity and avidity of antibodies. Using this approach, we found that most combinations produced killing percentages higher than each individual serum, although the increase was not additive. Sera with relatively high antibody titers were obtained, and this is because of the immunization scheme and bacterial concentration used, recalling that the rabbits were immunized in the absence of adjuvant.

Antibodies induced against whole cells of $N$. lactamica showed a high degree of cross-reactivity with $N$. meningitidis and commensal strains in ELISA. Meanwhile, the titers of bactericidal activity were not significant in sera raised against $N$. lactamica whole cells. Only rabbit serum raised against the $N$. meningitidis strain showed strong in-vitro bactericidal activity towards these strains. Furthermore, highavidity $\lg \mathrm{G}$ antibodies were produced when we performed a modified immunoglobulin $\mathrm{G}$ (IgG) ELISA, although a significant correlation was not found between ELISA titers and bactericidal activities. Meanwhile, the induction of $\operatorname{lgG}$ antibodies of high avidity obtained for all the sera against that test strain suggests that other mechanisms are involved such as opsonophagocytosis [5]. These results support the hypothesis that successive colonizations by $N$. lactamica can induce an effective immunity against meningitidis even if each commensal strain does not elicit a significant protection on its own, and it is possible that antigenic proteins on the surface of $N$. lactamica are able to prime the immune system in such way that it is immediately triggered upon the presence of $N$. meningitidis. Similar results were obtained by Sanchez et al. [4] who studied the induction of memory- 
driven responses against Neisseria meningitidis by priming with Neisseria lactamica using a mouse model.

Low antibody response to bactericidal activity (principally with $N$. lactamica) as opposed to the high IgG antibodies titers obtained and high-avidity antibodies, can be explained as follows: the protection is independent of PorA - a surface protein that is immunodominant and highly immunogenic, but also highly variable - and may target crossreactive antigens conserved between Neisseria species and meningococcal strains. Other mechanisms such as opsonophagocytosis activity may also be involved in the protective antibody response, demonstrating that bactericidal activity may not be the only predictor of protection for protein-based meningococcal vaccines [7].

In addition, bactericidal activity can be improved if we prime the immune system with $N$. lactamica followed by a boost with meningococcal, indicating that $N$. lactamica can prime the immune system for a cross-reactive response to $N$. meningitidis. In this study, we can see that after the immunization scheme in which the rabbits received two doses of $N$. lactamica followed by two doses of $N$. meningitidis, the titers of bactericidal activity obtained were improved, but it was not satisfactory. This suggests that the administration of more doses of $N$. lactamica in the immunization scheme are necessary. However, the immunization protocol used in this study was satisfactory in obtaining high titers of $\operatorname{IgG}$ antibodies in ELISA and high-avidity IgG antibodies. Other studies are underway in our laboratory.

As we can see, the contact of $N$. lactamica or Neisseria species must be also necessary to raise immunity against specific meningococcal antigens such as PorA and, possibly, some other antigens not yet identified responsible for bactericidal activity. In this respect, it is interesting to point out that some cross-reactive antigens may not be exposed in the same way in different strains, which requires a more in-depth study, not only of the antigens chosen for vaccine designs, but also of the strains used for their development or testing.

In conclusion, the rabbits immunized intranasally with whole cells of $N$. meningitidis or strains of Neisseria species in our study showed the following points. Whole cells of the bacterial strains used in this study when administered intranasally induced systemic immunity, as seen principally by high levels of $\operatorname{IgG}$ antibodies in the sera.Antibody responses against a broad range of outer membrane antigens were induced, including both outer membrane proteins and LOS, and major responses were elicited against 50-60, 70-80 and $180 \mathrm{kDa}$ antigens in immunoblotting.

Recent studies demonstrated that components present in the outer membrane of $N$. lactamica are immunogenic and present immunogenicity as in the case of PorB to a heterologous antigen [27]. These antigens are being studieds by several research groups for choice of antigens in the preparation of a vaccine against $N$. meningitidis using a universal antigen $[28,29]$.

This is the first study showing the importance of crossreactive antigens with Neisseria species bacteria using the rabbit nasal route as a model. The cross-reactive antigens against these pathogens have been demonstrated previously in other models. The results should help to determine the variability of the antigens present in commensal strains and their applicability in future vaccine designs.

\section{AUTHOR SUMMARY}

Serogroup B strain are responsible for meningococcal disease in Brazil and no suitable vaccine is available that confers universal protection against all serogroup B strains. Neisseria lactamica shares many antigens with the meningococcus, except capsule and the surface protein PorA. Many of these antigens are thought to be responsible for providing cross-protective immunity to meningococcal disease. An alternative approach to a vaccine for serogroup B meningococcal disease has been the development of an NOMV vaccine based on the commensal $N$. lactamica, a close relative of $N$. meningitidis. $N$. lactamica colonizes the human nasopharynx, particularly in infancy and childhood, and its presence has been implicated. Immunological and epidemiological evidence suggests that carriage of $N$. lactamica contributes to the age-related development of natural immunity against meningococcal disease, most probably due to the development of antibodies against the many surface structures in common with $N$. meningitidis. We used a homologous and heterologous prime/booster nasal immunization with Neisseria species in rabbit.

\section{ACKNOWLEDGEMENTS}

We thank Dr. W. D. Zollinger of the Walter Reed Army Institute of Research, Maryland for supplying the monoclonals used in serotyping. Strains of N. lactamica, N. sicca and $N$. meningitidis obtained from the oropharynx of carriers were kindly provided by Dr. Ferreirós of the Department of Microbiology and Parasitology of Departament of Microbiology, Faculty of Pharmacy, University of Santiago of Compostela. The reference center for N.meningitidis Adolfo Lutz Institute supplied $N$. lactamica, $N$. subflava, $N$. perflava, $N$. elongata, $N$. sicca and N. mucosa strains of Brazil. Claudia F Tunes and Aline S Ferraz are Master's students under the supervision of EN De Gaspari. Monica CG Scola was a research technician that managed the growth and control of the $N$. meningitidis strains used in this study. This work was supported by FAPESP and CNPq grants awarded to Dr. Elizabeth N. De Gaspari for several projects in the area since 1988. We are also grateful to Dr. A. Leyva for English editing of the manuscript.

\section{ABBREVIATIONS}

Neisseria $=\mathrm{N}$. meningitidis $\mathrm{c}$-carrier-isolated

meningitidis $\mathrm{c}$

N. sicca $=$ Neisseria sicca

$N$. lactamica $=$ Neisseria lactamica

$N$. meningitidis $=$ Neisseria meningitidis

$N$. flavencis $=$ Neisseriaflavencis

$N$. elongata $=$ Neisseria elongata

N. mucosa = Neisseria mucosa

LOS $=$ Lipooligosaccharide

OMP $=$ Outer Membrane Protein 


\begin{tabular}{|c|c|c|}
\hline $\operatorname{IgG}$ & $=$ & $\begin{array}{l}\text { Immunoglobulin "heavy" chains } \\
\text { gamma }\end{array}$ \\
\hline $\operatorname{Ig} \mathrm{A}$ & $=$ & $\begin{array}{l}\text { Immunoglobulin "heavy" chains } \\
\text { alpha }\end{array}$ \\
\hline Opa & $=$ & $\begin{array}{l}\text { Opacity-associated outer mem- } \\
\text { brane proteins }\end{array}$ \\
\hline Tbps & $=$ & Transferring-binding proteins \\
\hline FbpA & $=$ & Ferric binding proteins $\mathrm{A}$ \\
\hline OMVs & $=$ & Outer Membrane Vesicles \\
\hline PorA & $=$ & Class 1 protein of $N$. meningitidis \\
\hline PorB & $=$ & $\begin{array}{l}\text { Class } 2 \text { or } 3 \text { protein of } N \text {. menin- } \\
\text { gitidis }\end{array}$ \\
\hline SBA & $=$ & Serum Bactericidal Activity \\
\hline TSB & $=$ & Tryptic Soy Broth \\
\hline PBS & $=$ & Phosphate-Buffered Saline \\
\hline $\mathrm{LiCl}$ & $=$ & Lithium chloride \\
\hline SDS-PAGE & $=$ & $\begin{array}{l}\text { Sodium Dodecyl Sulfate poly- } \\
\text { acrylamide gel }\end{array}$ \\
\hline HRP & $=$ & Horseradish Peroxidase \\
\hline $\mathrm{AEC}$ & $=$ & 3-Amino-9-ethylcarbazole \\
\hline TMB & $=$ & $3,3^{\prime}, 5,5^{\prime}-$ Tetramethylbenzidine \\
\hline BHI & $=$ & Brain Heart Infusion Agar \\
\hline $\mathrm{VCN}$ & $=$ & $\begin{array}{l}\text { Vancomycin, Colistin and Nys- } \\
\text { tatin }\end{array}$ \\
\hline $\mathrm{CFU}$ & $=$ & Colonic Forming Units \\
\hline ELISA & $=$ & $\begin{array}{l}\text { Enzyme-linked immunosorbent } \\
\text { assay }\end{array}$ \\
\hline $\mathrm{SCN}$ & $=$ & Ammonium thiocyanate \\
\hline NL & $=$ & Neisseria lactamica \\
\hline NM & $=$ & Neisseria meningitidis \\
\hline NS & $=$ & Neisseria sicca \\
\hline $\mathrm{Nc}$ & $=$ & N. meningitidis isolated-carrier \\
\hline MW & $=$ & Molecular weight \\
\hline
\end{tabular}

\section{REFERENCES}

[1] Fisseha, M.; Chen, P.; Brandt, B.; Kijek, T.; Moran, E.; Zollinger, W. Infect. Immun., 2005, 73, 4070-80.
[2] Shoemaker, D.R.; Saunders, N.B.; Brandt, B.L.; Moran, E.E.; Laclair, A.D.; Zollinger, W.D. Infect. Immun., 2005, 73, 5031-8.

[3] Sánchez, S.; Troncoso, G.; Ferreirós, C.M.; Criado, M.T. Vaccine, 2001, 14, 390-8.

[4] Sánchez, S.; Troncoso, G.; Criado, M.T.; Ferreirós, C. Vaccine, 2002, 20, 2957-63.

[5] Gorringe, A.; Halliwell, D.; Matheson, M.; Reddin, K.; Finney, M.; Hudson, M. Vaccine, 2005, 18, 2210-3.

[6] Troncoso, G.; Sánchez, S.; Moreda, M.; Criado, M.T.; Ferreirós, C.M. FEMS Immunol. Med. Microbiol., 2000, 27, 103-9.

[7] Gorringe, A.R. Expert Rev. Vaccines, 2005, 4, 373-9.

[8] Sardiñas, G.; Reddin, K.; Pajon, R.; Gorringe, A. Vaccine, 2006, 24, 206-14.

[9] Oliver, K.J.; Reddin, K.M.; Bracegirdle, P.; Hudson, M.J.; Borrow, R.; Feavers, I.M.; Robinson, A.; Cartwright, K.; Gorringe, A.R. Infect. Immun., 2002, 70, 3621-6.

[10] Drabick, J.J.; Brandt, B.L.; Moran, E.E.; Saunders, N.B.; Shoemaker, D.R.; Zollinger, W.D. Vaccine, 1999, 20, 160-72.

[11] Welsch, J.A.; Moe, G.R.; Rossi, R.; Adu-Bobie, J.; Rappuoli, R.; Granoff, D.M. J. Infect. Dis., 2003, 188, 1730-40.

[12] O'dwyer, C.A.; Reddin, K.; Martin, D.; Taylor, S.C.; Gorringe, A.R.; Hudson,; M.J.; Brodeur, B.R.;Langford, P.R.; Kroll, J.S. Infect. Immun., 2004, 72, 6511-8.

[13] Li, Y.; Zhang, Q.; Winterbotham, M.; Mowe, E.; Gorringe, A.; Tang, C.M. Infect. Immun., 2006, 74, 6348-55.

[14] Gómez, J.A.; Criado, M.T.; Ferreirós, C.M. FEMS Immunol. Med. Microbiol., 1998, 20, 79-86.

[15] Lowry, O.H.; Rosebrough, J.N.; Farr, L.; Randall, R.J. J. Biol. Chem., 1951, 193, 265-75.

[16] De Gaspari, E.N. Braz. J. Infect. Dis., 2000, 19, 445-53.

[17] Laemmli, U. K. Nature, 1970, 227, 680-5.

[18] Towbin, H.; Staehelin, T.; Gordon, J. Proc. Natl. Acad. Sci. USA 1979; 76, 4350-4.

[19] Abdillahi, H.; Poolman, J.T. J. Med. Microbiol., 1988, 26, 177-80.

[20] Mandrell, R.E.; Azmi, F.H.; Granoff, D.M. J. Infect. Dis., 1995, 172, 1279-89.

[[21] Maslanka, S.E.; Gheesling, L.L.; Libutti, D.E.; Donaldson, K.B.; Harakeh, H.S.; Dykes, J.K.; Arhin, F.F.; Devi, S.J.; Frasch, C.E.; Huang, J.C.; Kriz-Kuzemenska, P.; Lemmon, R.D.; Lorange, M.; Peeters, C.C.; Quataert, S.; Tai, J.Y.; Carlone, G.M. Clin. Diagn. Lab. Immunol., 1997, 4, 156-67.

[22] Granoff, D.M.; Maslanka, S.E.; Carlone, G.M.; Plikaytis, B.D.; Santos, G.F.; Mokatrin, A.; Raff, H.V. Clin. Diagn. Lab. Immunol., 1998, 5, 479-85.

[23] Chackerian, B.; Lowy, D.R.; Schiller, J.T. J. Clin. Invest., 2001, $108,415-23$.

[24] Suckow, M.A.; Bowersock, T.L.; Nielsen, K.; Grigdesby, C.F. Lab. Anim., 1996, 30, 120-6.

[25] Lu, Y,S.; Lai, W.C.; Pakes, S.P.; Nie, L.C. Infect. Immun., 1991, 59, 172-80.

[26] Arenas, J.; Abel, A.; Sánchez, S.; Alcalá, B.; Criado, M.T.; Ferreirós, C.M. Int. Microbiol., 2006, 9, 273-80.

[27] Liu, X.; Wetzler, L.M.; Massari, P. Vaccine, 2008, 26, 786-96.

[28] Finney, M.; Vaughan, T.; Taylor, S.; Hudson, M.J.; Pratt, C.; Wheeler, J.X.; Vipond, C.; Feavers, I.; Jones, C.; Findlow, J.; Borrow, R.; Gorringe. Hum. Vaccine, 2008, 4, 23-30.

[29] Holst, J. Hum. Vaccine, 2007, 290-4. 